\title{
A Lesson Learned from a Rare Case of Missing Guide Wire
}

\author{
Ng Kwang How, Anwar Irawan Ruhani \\ Department of Cardiology, Hospital Tengku Ampuan Afzan, Kuantan, Pahang, Malaysia \\ Email: kwang_how@yahoo.com
}

How to cite this paper: How, N.K. and Ruhani, A.I. (2022) A Lesson Learned from a Rare Case of Missing Guide Wire. Open Access Library Journal, 9: e8334. https://doi.org/10.4236/oalib.1108334

Received: December 28, 2021

Accepted: January 22, 2022

Published: January 25, 2022

Copyright ( 2022 by author(s) and Open Access Library Inc.

This work is licensed under the Creative Commons Attribution International License (CC BY 4.0).

http://creativecommons.org/licenses/by/4.0/

\section{(c) (i) Open Access}

\begin{abstract}
Central venous catheterization via the Seldinger technique is a minimally invasive procedure which is increasingly and widely performed. It is associated with immediate and delayed complications which might cause significant morbidity and mortality. The retained guidewire is a rare, potentially serious but completely avoidable complication. We reported a 74-year-old gentleman who presented with upper gastrointestinal bleeding and was successfully resuscitated. Central venous catheterization was done but complicated with an inadvertently retained guidewire. Endovascular retrieval of the retained guidewire was successfully performed under fluoroscopic guidance and patient was subsequently discharged well. We also described its predisposing factors, preventive strategies and its successful management. This case highlights the importance of correct technique, preventive strategies and timely rectification of medical errors.
\end{abstract}

\section{Subject Areas}

General Medicine, Cardiology

\section{Keywords}

Central Venous Catheterization, Retained Guide Wire, Endovascular Retrieval

\section{Introduction}

As the technology of minimally invasive procedures advanced, procedures using intravascular objects have been increasing rapidly. Central venous catheterization (CVC) via the Seldinger technique is one of such procedures, commonly performed in critically ill patients in various departments. It is usually indicated for delivery of certain medications, prolonged intravenous therapies, parenteral 
nutrition, hemodialysis and difficult peripheral venous access. The common cannulation sites are femoral, jugular, subclavian and brachial veins. The increasing usage is associated with an increased risk of procedure-related complications. Correct operative skills, expert supervision, and attention to all details are needed to prevent complications. Common complications associated with CVC placement are infection, arterial puncture, hematoma, pneumothorax or vessel injury [1] [2]. Inadvertently retained guidewire is a rare complication, and can be recognized immediately during the procedure or with delay [1]. We presented a rare case of inadvertently retained guidewire following central venous catheterization, and described its predisposing factors, preventive strategies, endovascular retrieval and its successful outcome.

\section{Case Presentation}

A 74-year-old gentleman with underlying diabetes, hypertension and peptic ulcer disease presented with anemic symptoms, hematemesis and melena. He was intubated for impending respiratory collapse upon arrival at the emergency department. His haemoglobin was $4.4 \mathrm{~g} / \mathrm{dL}$. Immediate oesophagogastroduodenoscopy showed a Forest $1 \mathrm{~b}$ ulcer at the gastro-oesophageal junction. Injection of adrenaline 1:10,000 was performed. He was diagnosed to have severe anemia secondary to upper gastrointestinal bleeding. Resuscitation with intravenous fluid, blood transfusion, inotropic support and pantoprazole infusion were done. The central venous catheterization was performed under aseptic precautions at the right internal jugular vein via Seldinger technique. No difficulties during the procedure or immediate complications. Post-procedural chest X-ray showed a retained guide wire in the chest. Plain abdominal radiography confirmed the position of the guidewire's tip, which had reached the right iliac vein (Figure 1). He was referred to cardiology team for endovascular guidewire retrieval after multi-disciplinary team discussion. As guidewire is a radio-opaque object, retrieval was done under fluoroscopic guidance with electrocardiogram and hemodynamic monitoring. Multiple loop snare was passed through a guiding catheter via left femoral venous approach. It captured the wire's distal end and retracted gradually. However the wire was dislodged when it reached the left iliac vein. Snaring was re-attempted and finally the whole wire was removed successfully (Figure 2). The patient was adequately heparinized throughout the procedure. Subsequently he had recovered from his illness and was discharged well.

\section{Diagnosis}

The retained guidewire following central venous catheterization is usually diagnosed by clinical suspicion or established incidentally after the routine postprocedural radiography [1] [3]. Post-procedural radiography can also confirm the location of the retained guidewire and detect other CVC placement related complications, for example, pneumothorax or hemothorax if CVC is inserted at subclavian vein or internal jugular vein. 


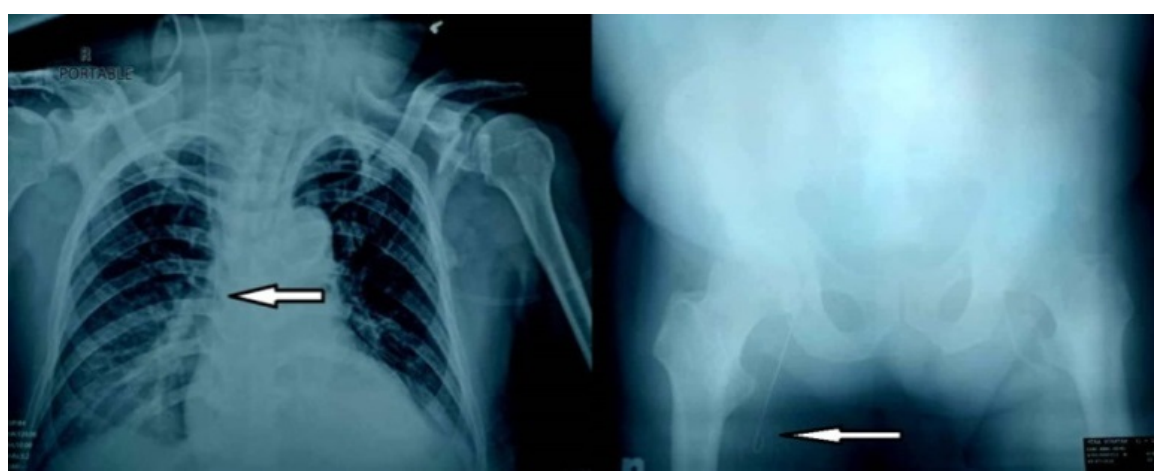

(a)

(b)

Figure 1. (a) Chest X-ray showed a retained guide wire; (b) Abdominal radiography confirmed the position of the guidewire's proximal tip.

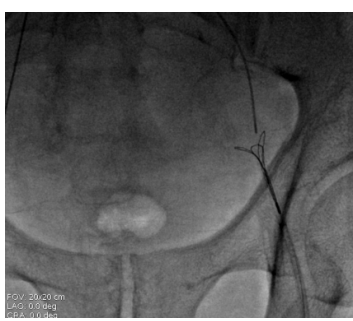

(a)

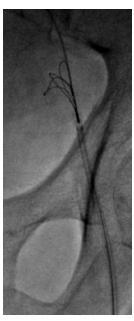

(b)

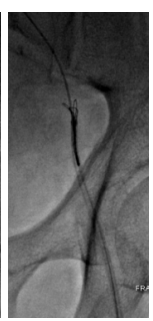

(c)

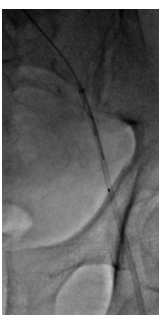

(d)

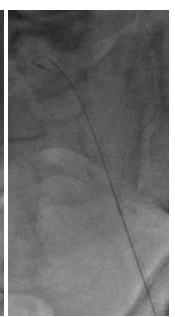

(e)

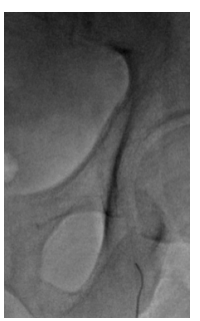

(f)

Figure 2. Progression of the snaring. (a) Multiple loop snaring was approaching the retained guidewire's distal end; (b, c) Capturing the wire; (d) Successfully captured and retracted into the guiding catheter; (e, f) Arrows showed the movement of the wire's proximal tip during the retrieval process.

\section{Discussion}

Retained guide wire is a rare but completely preventable complication. Although it is rare, minimally invasive procedures require higher awareness of retained foreign object due to its nature of reduced operative field's visibility. It also raises the concerns of patient safety, operator responsibility and potential medicolegal consequences. Migration of a broken guide wire fragment in the circulation might cause arrhythmias, vascular damage, thrombosis and embolism. Embolism from the fragments of catheters or guide wires can be fatal up to $20 \%$ [4]. However, a complete guide wire loss into the circulation might be asymptomatic and may remain unnoticed for a significant period of time [5] [6]. The signs are the wire is missing, resistance to injection or poor venous backflow from the distal lumen, or wire is detected on radiograph [1]. Possible causes are operator inexperience or fatigue, inadequate supervision, inattention and poor lighting. Every single step of central venous cannulation is important as it might cause complications. All operators should be fully aware of the risks and complications and continuous attention during the procedure is vital. Establishing a standard of practice policy is necessary to prevent the potential unnecessary morbidity, mortality and additional invasive and costly retrieval procedures. It included checking for wire defects before insertion, ensuring the wire protrudes from the 
proximal end of the catheter and gripping it firmly before advancing the catheter, rechecking for damage and repositioning the introducer when encountering resistance, counting needles and wires, checking for complete wire removal after the procedure, and continuous medical training for technique improvement [1] [7]. An immediate post-procedural radiograph is important to confirm the position of the catheter and to detect complications. It is important to remove the retained guidewire with appropriate tools and expertise, as quickly and completely as possible [8]. Percutaneous retrieval of intravascular foreign bodies was first introduced in 1964 by Thomas et al., and has become a preferred approach for intravascular objects removal since then [9]. Endovascular retrieval's options are loop snare, balloon catheter, basket retriever, and grasping forceps. The standard method is the loop snare via femoral vein under radiographic guidance, which has higher success rate and lower complications [10] [11]. Retrieval of retained guide wire has a success rate of $90 \%$ [12]. The use of endovascular forceps or Dormier baskets increases the risk of endovascular trauma [13]. Patient should be adequately heparinized during the intervention [13]. If heparinization is contraindicated, careful surgical exploration and extraction should be considered [7].

\section{Conclusion}

Our intention of reporting this inadvertently retained guide wire and its management is to emphasize the importance of correct technique, preventive strategies, risks awareness, timely rectification of medical errors, and effective multidisciplinary team communication.

\section{Conflicts of Interest}

The authors declare no conflicts of interest regarding the publication of this paper.

\section{References}

[1] Srivastav, R., Yadav, V., Sharma, D. and Yadav, V. (2013) Loss of Guide Wire: A Lesson Learnt Review of Literature. Journal of Surgical Technique and Case Report, 5, 78-81. https://doi.org/10.4103/2006-8808.128732

[2] Wolf, F., Schernthaner, R.E., Dirisamer, A., Schoder, M., Funovics, M., Kettenbach, J., et al. (2008) Endovascular Management of Lost or Mis-Placed Intravascular Objects: Experiences of 12 Years. CardioVascular and Interventional Radiology, 31, 563-568. https://doi.org/10.1007/s00270-007-9201-8

[3] Guo, H., Peng, F. and Ueda, T. (2006) Loss of the Guide Wire: A Case Report. Circulation Journal, 70, 1520-1522. https://doi.org/10.1253/circj.70.1520

[4] Heberer, M., Moser, J., Durig, M. and Harder, F. (1984) Prospective Study of Complications of Central Venous Catheters. Transfusion Medicine and Hemotherapy, 11, 254-261. https://doi.org/10.1159/000221671

[5] Abuhasna, S., Abdallah, D. and ur Rahman, M. (2011) The Forgotten Guide Wire: A Rare Complication of Hemodialysis Catheter Insertion. Journal of Clinical Imaging Science, 1, Article No. 40. https://doi.org/10.4103/2156-7514.83397 
[6] Wadehra, A., Ganjoo, P. and Tandon, M.S. (2010) Guide Wire Loss during Central Venous Cannulation. Indian Journal of Anaesthesia, 54, 587-588. https://doi.org/10.4103/0019-5049.72665

[7] Schummer, W., Schummer, C., Gaser, E. and Bartunek, R. (2002) Loss of the Guide Wire: Mishap or Blunder? British Journal of Anaesthesia, 88, 144-146. https://doi.org/10.1093/bja/88.1.144

[8] Michaelis, G. and Biscoping, J. (2000) Clinical Significance and Effects of Foreign Body Embolism during the Use of Central Venous Catheters. Anasthesiol Intensivmed Notfallmed Schmerzther, 35, 137-140. https://doi.org/10.1055/s-2000-8747

[9] Thomas, J., Sinclair-Smith, B., Bloomfield, D. and Davachi, A. (1964) Non-Suurgicalretrieval of a Broken Segment of Steel Spring Guide from the Right Atrium and Inferior Vena Cava. Circulation, 30, 106-108.

https://doi.org/10.1161/01.CIR.30.1.106

[10] Cheng, C.C., Tsai, T.N., Yang, C.C. and Han, C.L. (2009) Percutaneous Retrieval of Dislodged Totally Implantable Central Venous Access System in 92 Cases: Experience in a Single Hospital. European Journal of Radiology, 69, 346-350.

https://doi.org/10.1016/j.ejrad.2007.09.034

[11] Egglin, T.K., Dickey, K.W., Rosenblatt, M. and Pollak, J.S. (1995) Retrieval of Intravascular Foreign Bodies: Experience in 32 Cases. American Journal of Roentgenology, 164, 1259-1264. https://doi.org/10.2214/ajr.164.5.7717243

[12] Dondelinger, R.F., Lepoutre, B. and Kurdziel, J.C. (1991) Percutaneous Vascular Foreign Body Retrieval: Experience of an 11-Year Period. European Journal of Radiology, 12, 4-10. https://doi.org/10.1016/0720-048X(91)90124-E

[13] Kessel, D. and Robertson, I. (2000) Interventional Radiology-A Survival Guide. Churchill-Livingstone, London, 225-229. 